\title{
Introduction: Overall vision of ethics in nanotechnology developments
}

\section{Book's objective}

The new and emerging nanotechnology is remaking the world at an alarmingly rapid pace with many applications in a wide variety of fields ranging from health care over industrial products to crime prevention and defense. Despite the many impactful benefits of nanotechnology, such as the cultivation of new organs for patients, there are potential hazards and risks involved, such as the toxicity of certain nanoparticles that could cross the blood-brain barrier. Nanotechnology, it should be clear, has the potential to profoundly change society for better or for worse.

The societal implications of nanotechnology encompass many ethical issues such as the violation of privacy, the violation of a person's autonomy, environmental pollution, economic abuse, security, and global justice (between developed and developing countries).

The biggest question we are facing is how we should deal with uncertainty and risk in this emerging technology? Uncertainty is one of the major obstacles to the commercialization of nanotechnology - uncertainty about the risks to health and environment and uncertainty about how the governments might regulate nanotechnology in the future. There is an urgent need for answers both in the realm of science and in the realm of law and regulation. In the absence of scientific clarity about the potential health and environment effects of exposure to nanoparticles, the policymakers must be provided with guidance on how to deal with hazards, risks and controls.

Nanotechnology will affect everyone; thus, all members of society should have a voice in its development and commercialization. The book focuses on the main societal and ethical issues that arise from the development of nanotechnology. The result is a clear set of questions and solutions proposed by leading researchers working in a variety of fields such as applied ethics, bioethics, ethics of science, ethics of technology, and business ethics.

There is an urgent need for constructive interaction between the natural sciences (physics, chemistry, mathematics, biology, and engineering) and the human sciences (philosophy, law, sociology, and ethics), with the aim of formulating ethical standards for the development of nanotechnology. This book hopes to be an important step in that direction.

This book presents an overview of new and emerging nanotechnologies and their societal and ethical implications. To support sustainable, ethical, and economic nanotechnological development, it is imperative to educate all nanotechnology scientists, engineers, and stakeholders about the long-term benefits, limitations, and risks of nanotechnology. In the introduction, we provide the reader with an insight into the multiple societal and ethical questions raised by nanotechnology. 


\section{Convergence of natural and human sciences in nanotechnology}

Nanotechnology is a popular term in these days, which is associated with multiple uses and potential applications in the future. It is a dynamic multidisciplinary field. The applications are constantly extending to various areas, and their implementations are impressive. In fact, the main engineer/alchemist offering nanoscale materials appears to be life's unstoppable force, our mother nature. In ancient times, certain practices created nanoparticles through the customary procedures. One of the most acclaimed ancient applications is the Lycurgus cup, which was created from nanoparticles of gold and silver that were inserted in the glass. However, these were not recognized as nanosystems or nanoparticles until the twentieth century.

One nanometer is basically one billionth of a meter, or $10^{-9}$ of a meter. A newspaper sheet is around 100,000 nm wide, in contrast. Nanotechnology - as we know it was introduced in 1959 in a talk delivered by Richard Feynman, "There's plenty of room at the bottom." Nevertheless, until 1974, when a Japanese physicist Norio Taniguchi invented and described the word "nanotechnology," the real phrase "nanotechnology" was not coined. Thereafter, the development of scientific instruments such as the scanning tunneling microscope (1981) and the atomic force microscope (1986) generated the impetus for the further development of contemporary nanotechnology. Scientists discovered that atoms and molecules behave differently at the nanoscale. As a result, experts believe that nanotechnology has potential applications in a broad variety of fields with significant consequences for human well-being, the atmosphere, biodiversity, and national security. Although plenty of us do not realize the incredible effect it might have and - to a certain extent - is already having on our everyday lives. A promising indication is the growing amount of capital that policymakers are investing into improving such innovations worldwide.

With the advent of the fourth industrial revolution encompassing robotics, artificial intelligence, machine learning, Internet of things, 3D printing, and so on, the world of nanotechnology will change our lives and our society dramatically. There will be a great number of applications that, at present, we cannot even conceive.

Some of the important examples of applications include the following:

\section{In medicine:}

- Nanoparticle-based vaccines may one day provide permanent immunity to the common cold and influenza.

- Artificial replacements for body tissues such as the skin, muscle, tendon, and even organs can be produced by nanoscale fabrication, mimicking natural processes.

- Nanoparticles in pharmaceutical products will smartly deliver chemotherapy drugs to specific cells, such as cancer cells. 


\section{In consumer product:}

- Filters for producing clean drinking water will remove all viruses and bacteria.

- Nanoparticles used in food packaging will reduce UV exposure to prolong shelf life.

- Faster, smaller, and more powerful computers will consume far less power, with biodegradable longer lasting batteries.

\section{In industrial applications:}

- Nano-strengthened materials will produce lightweight alloys for cars, reducing fuels.

- Tires will be fabricated with a better grip in wet conditions.

- Electrode materials for rechargeable batteries will be greatly enhanced through nanotechnology, reducing weight and improving performance in the next generation of hybrid and electric vehicles.

History shows that new science and technology is often met by society with unrest. Prominent examples include the development of synthetic chemicals and nuclear power in the mid-twentieth century, and biotech and genomics in the twentieth century. The same goes for nanotechnology and the potential threats it raises. Therefore, there is an urgent need for a better understanding of the safety aspects of nanotechnology applications. For the nanomaterials, numerous toxicological tests have shown that nanoparticles can damage both humans and the ecosystem. Many argue that we should apply to the precautionary principle to engineered nanoproducts and particles. An expert study published by the Federal Ethics Committee on Non-Human Biotechnology (ECNH) concluded that the presumption of proof of the harmlessness of synthetic nanoparticles will be the duty of the producers because there are good reasons to believe that such particles may seriously endanger humans and the atmosphere under some circumstances. Moreover, given the unpredictability of the future developments in nanotechnology, governmental authorities and consumers are faced with unknown risks.

Nanotechnology, it should be clear, has the power to revolutionize human lives and transform human societies. We must ask ourselves, what kind of world are we creating? The development of nanoscience and nanotechnology should not be left to engineers and nanoscientists alone. It is of utmost importance that experts in the social sciences and humanities and the public at large are involved. Developments in nanotechnology should be transparently communicated and closely monitored. For this purpose, scientists, engineers, and industrial players should work closely together with ethicists and social scientists and the public should be informed and consulted.

At present, such a multidisciplinary and ongoing dialogue on the ethical concerns surrounding nanotechnology is, for the most part, lacking. This book hopes to contribute to this urgent task. It addresses the ethical concerns raised by nanotechnology from various perspectives: from a scientific, social, and philosophical point of 
view. Chapters have been written by experts from various countries across the world, including the United States, Japan, China, Israel, India, Australia, Canada, and European countries, proposing different strategies but reaching the same conclusion that there is an urgent need for ethical guidelines as well as regulations to ensure safe and healthy production and use of nanoproducts. It is also clear that these regulations and guidelines should have a worldwide dimension.

The book is divided into two volumes. The purpose of this book is to discuss the theoretical and philosophical/ethical aspects of nanotechnology.

The first volume, entitled Emerging Technologies Aspects, deals with the important ethical questions of the implications of nanotechnology on human well-being and health as well as the environment. It comprises 17 chapters divided into 3 sections. The chapters are written by the leading scientists and philosophers in their respective fields. The first section focuses on nanotechnology and its implications for philosophy, ethics, and society. It comprises two chapters: the first chapter covers the orientation of nanoethics, whereas the second chapter discusses the need for nanotechnology to mitigate technological difficulties. This is followed by six chapters in the second section that address ethical concerns in nanoscience. Chapters 3-9 discuss about applications of nanotechnology and their future, along with the development of guidelines. Chapters 10-14 in Section 3 deal with ethical reflections in health care and the environment. This includes nanosafety of biomedical nanomaterials, nanomedicine, and nanodrugs. The chapters are authored by experts in these fields, and they have discussed their own researches as well as the developments in their fields of interest. It is our sincere hope that this multiauthored book covering the various aspects of nanotechnology and ethics will be welcomed by the scientific community and philosophers alike. We sincerely hope that the book will assist and enrich readers to understand the ethical challenges of nanomaterials and the discussed solutions to the safe use of nanomaterials.

The second volume, entitled Social Sciences and Philosophical Aspects, addresses the ethical and social implications of nanotechnology. It comprises 16 chapters. The book is divided into four sections. The first section has one chapter dedicated to revitalized vision on ethics and safety for technological implications. The second section has five chapters explaining on ethical concerns in nanotechnology developments. The first chapter in this section, Chapter 2, deals with the ethics, and regulatory and governance of nanotechnology in agriculture and food. The subsequent chapters cover the ethical issues for balanced and inclusive nanotechnology in various areas, including nanocosmetics and pharma developments, risk screening tools for engineered nanomaterials, and human health effect impacts on environment and society, respectively. The last chapter of this section presents views about nanoethics from a developing country point of view. Third section is about the philosophy of nanotechnology and nanoethics. It discusses the theological approach toward nanotechnology. Fourth section is a special section that deals with ethical recommendations for promising technology. 
The book also highlights perception of nanotechnology and ethical concerns from different angles and proposes a holistic approach toward the perceived dangers of nanotechnology.

We believe this book will be equally relevant to scientists and engineers employed in the area of nanotechnology and philosophers involved in nano- or any technology-related ethics. The text is structured in such a manner that each chapter stands on its own and can be read independently.

We express our sincere thanks to Ms Ute Skambraks for her kind help in publication process. Sincere thanks to Dr Karin Sora for continually supporting us through the publication house. We express our sincere gratitude to all the writers who made diligent efforts to write chapters during the time of the COVID-19 pandemic. We are grateful to all the eminent persons: Mihail C. Roco - National Science Foundation and National Nanotechnology Initiative; Peter Brabeck-Lemathe - Former President NESTLE; Shailendra Saraf - Vice President, Pharmacy Council of India; and Federico Mayor Zaragosa - Former President of UNESCO from respectable authorities for the excellent foreword to this book. We appreciate our respective families, for without their continuous support this work could not have been completed.

Marcel Van de Voorde and Gunjan Jeswani, book editors 
